\title{
Cuidado de enfermagem ao cliente com hipertensão: uma revisão bibliográfica
}

\author{
Nursing care to the client with hypertension: a bibliographic review \\ Atención de enfermería al cliente con hipertensión: una revisión bibliográfica
}

\section{Denizielle de Jesus Moreira Moura', Sara Taciana Firmino Bezerra', Thereza Maria Magalhães Moreira", Ana Virgínia de Melo Fialho"}

' Universidade Estadual do Ceará, Programa de Pós-Graduação em Cuidados Clínicos em Enfermagem e Saúde. (Mestranda) Fortaleza-CE, Brasil.

" Universidade Estadual do Ceará, Curso de Graduação em Enfermagem, Programa de Pós-Graduação em Cuidados Clínicos em Enfermagem e Saúde. Fortaleza-CE, Brasil.

Submissão: 12/01/2010 Aprovação: 07/11/2010

\section{RESUMO}

O estudo objetivou identificar as práticas de cuidado de enfermagem ao hipertenso nas produções científicas dos últimos dez anos. Realizou-se um estudo bibliográfico, utilizando, por meio da BIREME, as bases LILACS, SciELO e BDENF, com seleção de trinta artigos. Os resultados estão expostos em quadros, tabelas e gráficos, onde predominou a consulta de enfermagem como prática mais utilizada nas produções, por meio da Sistematização da Assistência de Enfermagem e a Educação em Saúde com abordagens individuais, além de visitas domiciliárias na abordagem familiar. Acredita-se que a sistematização, na organização do cuidado, contribuirá sobremaneira à adesão ao tratamento anti-hipertensivo.

Descritores: Cuidados de Enfermagem; Hipertensão Arterial; Literatura de Revisão como Assunto.

\section{ABSTRACT}

This study aimed to identify the nursing care practices to the client with hypertension in the scientific production in the last ten years. It was carried out a bibliographic study, using in the BIREME, the LILACS, SciELO and BDENF's data basis, selecting thirty articles. The results were exposed in charts, tables and graphics, where prevails, in the literature analyzed, the nursing consultation as the more used practice, through the nursing attendance systematization and the Health Education with individual approaches, besides the realization of home visits with family approach. One believes that the systematization of nursing care will contribute in an expressive manner to the adhesion to the antihypertensive treatment.

Key words: Nursing care; Hypertension; Review literature as topic.

\section{RESUMEN}

Se objetivó identificar las prácticas del cuidado de enfermería al hipertenso en las producciones científicas de los últimos diez años. Se realizó una investigación bibliográfica, utilizándose vía BIREME, las bases LILACS, SciELO y BDENF, con la selección de treinta artículos. Los resultados están expuestos en cuadros, tablas y gráficos, en que predomina la consulta de enfermería, a través de la sistematización de la asistencia de enfermería y de la educación en salud con abordajes individuales, además de visitas en los sitios en el abordaje familiar. Se cree que la sistematización, en la organización de la atención, podrá contribuir para la adhesión al tratamiento anti hipertensión.

Descriptores: Atención de Enfermería; Hipertensión Arterial; Literatura de Revisión como Asunto. 


\section{INTRODUÇÃO}

Este estudo foi elaborado como parte das avaliações da disciplina "Pesquisa em Saúde e Metodologia Quantitativa" do Mestrado Acadêmico em Cuidados Clínicos em Saúde (CMACCLIS) da Universidade Estadual do Ceará - UECE. A temática cuidado de enfermagem ao cliente com hipertensão, por sua vez, foi escolhida por ser objeto de estudo das autoras e por sua relevância.

A Hipertensão Arterial Sistêmica (HAS), por seu perfil epidemiológico, tem sido estudada sob diferentes perspectivas, com o intuito de desenvolver uma prática profissional que proporcione sua prevenção ou controle por meio de mudanças de estilo de vida e/ou adesão ao tratamento farmacológico.

A hipertensão arterial é um dos principais fatores de risco para o desenvolvimento das doenças cardiovasculares, explicando $40 \%$ das mortes por acidente vascular cerebral e $25 \%$ por doença arterial coronariana ${ }^{(1)}$. No Brasil, há em torno de 17 milhões de pessoas com hipertensão, atingindo cerca de $35 \%$ da população a partir de 40 anos. É um fenômeno ascendente, cada vez mais precoce e que constitui grave problema de Saúde Pública no Brasil e no mundo ${ }^{(2)}$.

Tem como característica os níveis elevados da pressão arterial, sendo definida como pressão arterial sistólica igual ou maior que $140 \mathrm{mmHg}$ e/ou pressão arterial diastólica igual ou superior que $90 \mathrm{mmHg}$, em indivíduos sem uso de anti-hipertensivos $^{(2)}$. Entretanto, a delimitação do diagnóstico de hipertensão somente a partir das cifras pressóricas é arbitrária. Faz-se necessária ressaltar a importância da identificação dessa doença considerando níveis pressóricos, fatores de risco, lesões em órgãos-alvos e comorbidades associadas, visando prevenir equívocos ou danos às pessoas.

Embora se trate de uma doença crônico-degenerativa de fácil diagnóstico e com grande diversidade terapêutica, seu controle constitui um desafio aos pacientes em virtude das mudanças de estilo de vida necessárias; e aos profissionais de saúde, sobretudo aos enfermeiros, que têm sua ação pautada no cuidado contínuo a esses indivíduos. A esses profissionais, o maior desafio revela-se na necessidade de desenvolver estratégias com vistas a conduzir o indivíduo ao autocuidado e consequente adesão à terapêutica.

A adesão ao tratamento anti-hipertensivo é consolidada com a participação do cliente de forma ativa no seu plano terapêutico, não se constituindo em mero cumpridor de recomendações; ao contrário, é visto como sujeito do processo, assumindo com os profissionais de saúde, a responsabilidade pelo seu tratamento ${ }^{(3)}$.

A não adesão ao tratamento tem atingido aproximadamente $50 \%$ dos pacientes ${ }^{(4)}$ e envolve aspectos biológicos, psicológicos, culturais e sociais. A falta de controle da pressão arterial, mesmo em pacientes acompanhados em unidades de saúde, regularmente é freqüente.

Este fato tem contribuído para onerar os custos do Sistema de Saúde. No Brasil, ocorreram 150.000 internações por doenças cardiovasculares, com custo global de 475 milhões de reais( ${ }^{(5)}$. As consequências da não adesão ao tratamento da hipertensão arterial configuram-se como: aumento dos custos sociais com absenteísmo ao trabalho, licenças para tratamento de saúde, aposentadorias precoces por invalidez ${ }^{(6)}$, internações, óbitos e comprometimento da qualidade de vida dos grupos sociais mais vulneráveis ${ }^{(5)}$.

Assim, faz-se necessário o desenvolvimento de estratégias de cuidado que contemplem os diversos elementos envolvidos no processo de adoecimento da hipertensão arterial: as expressivas transformações na vida dos indivíduos nas esferas emocional, familiar, social e econômica, considerando que a maior parte constitui-se de usuários do Sistema Único de Saúde (SUS), dentre os quais estão embutidas dificuldades socioeconômicas e culturais que podem tornar-se empecilhos à adesão terapêutica adequada. Esse cuidado deve ser contextualizado às necessidades do indivíduo e permeado pela noção de autonomia, com vistas à produção de postura ativa na adesão.

Mesmo com o reconhecimento da Enfermagem na adesão ao tratamento, evidenciamos uma lacuna entre teoria e prática, uma vez que são elevados os índices de não adesão e a presença de complicações ${ }^{(7)}$.

A Enfermagem é uma ciência, cuja essência e especificidade é o cuidado ao ser humano, individualmente, na família ou na comunidade, desenvolvendo-o de forma autônoma e/ou, muitas vezes, em equipe; é uma profissão de ajuda com relações complexas e multifacetadas, composta por uma grande variedade de elementos; o seu cuidado transcende a dimensão biológica da pessoa, e tem como foco o ser que experiencia a doença, incluindo sua cultura, valores, crenças, modos de vida e sentimentos vinculados às suas necessidades de cuidado ${ }^{(8)}$.

Assim, torna-se urgente compreender como se dá a prática de enfermagem a essa clientela nos diversos cenários de atuação da saúde. A realização de pesquisas fornece subsídios para que os conhecimentos produzidos e divulgados sejam incorporados na assistência de enfermagem, melhorando a qualidade de vida dos pacientes portadores de hipertensão arterial. Assim, questionamo-nos: como o cuidado ao cliente com hipertensão tem sido abordado na produção científica da enfermagem nos últimos dez anos?

O objetivo do trabalho foi identificar, na produção do conhecimento, práticas de cuidados de enfermagem ao portador de hipertensão arterial na última década. Esperamos, a partir dos resultados, visualizar o cenário da produção científica de enfermagem sobre hipertensão arterial, promovendo análise sobre os diversos aspectos de cuidado descritos na literatura.

Tal análise torna-se importante no sentido de verificar a construção do conhecimento acerca da temática e identificar possíveis lacunas em seu arcabouço teórico-conceitual, seja em termos clínicos, conceitual ou empírico, incitando a estudos posteriores sobre o tema.

\section{METODOLOGIA}

Trata-se de um estudo bibliográfico do tipo revisão da literatura, acerca das práticas de cuidado de enfermagem a clientes com hipertensão, o que propicia a análise de um tema sob novo enfoque ou abordagem, inovando com conclusões críticas.

Para a seleção dos estudos, realizou-se levantamento de dados na Biblioteca Científica Eletrônica Online (SCIELO), na Literatura Latino-americana e do Caribe em Ciências da Saúde (LILACS) e no Banco de Dados em Enfermagem (BDENF). Foram critérios 
de inclusão: artigos publicados nas bases de dados citadas no período de 1998 a 2008; artigos com os seguintes descritores e marcadores booleanos: cuidados de enfermagem and hipertensão, enfermagem and hipertensão.

Após levantamento, identificou-se 105 publicações entre artigos de periódicos, dissertações e monografias. Foram excluídos os artigos que, embora tivessem tais descritores, não versavam especificamente sobre cuidados de enfermagem ao cliente com hipertensão (54), bem como aqueles que se repetiram ao compilar todas as bases de dados (21). Após leitura flutuante, selecionou-se 32 estudos, iniciando leituras exaustivas dos textos, fazendo uma síntese de cada artigo e categorizando os tipos de cuidados identificados.

Os dados foram tratados descritivamente com indicação de freqüências simples e percentuais, referentes ao ano e local de publicação, tipos de estudo, cenário do estudo e eixos temáticos. Foram apresentados em tabelas e gráficos e discutidos com base na literatura pertinente.

\section{RESULTADOS}

De acordo com os dados da Tabela 1, observamos que o quantitativo de trabalhos encontra-se diluído ao longo dos últimos dez anos. Entretanto, observamos a predominância de publicações sobre hipertensão nos anos de 2001 e 2002, com quatro e seis estudos, respectivamente. Tal fato pode estar relacionado ao grande número de trabalhos sobre o cuidado de enfermagem, publicados nos anais do $53^{\circ}$ e do $54^{\circ}$ Congressos Brasileiro de Enfermagem.

Tabela 1 - Descrição dos estudos sobre cuidados de enfermagem ao cliente com hipertensão, segundo ano de publicação e estado/país onde o estudo foi realizado. Fortaleza- CE, 2009.

\begin{tabular}{c|lll}
\hline Ano de publicação & N & $\begin{array}{c}\text { Estado/país onde o estudo } \\
\text { foi realizado }\end{array}$ & N \\
\hline 1998 & 2 & São Paulo & 8 \\
1999 & 3 & Ceará & 7 \\
2000 & 3 & Minas Gerais & 2 \\
2001 & 4 & Paraná & 2 \\
2002 & 6 & Rio Grande do Sul & 2 \\
2003 & 3 & Paraíba & 1 \\
2004 & 2 & Rio de Janeiro & 1 \\
2005 & 4 & México & 1 \\
2006 & 2 & Peru & 1 \\
2007 & 2 & Não informou* & 5 \\
2008 & 1 & & \\
\hline
\end{tabular}

Ainda na Tabela 1, evidenciamos que a maior parte dos estudos foi realizada no estado de São Paulo, com oito publicações, evidência que pode ser justificada pelo fato de ser este a principal força econômica-política do país, tendo também a maior concentração de instituições de ensino superior e de pós-graduação. Em segundo lugar, encontra-se o Ceará com sete trabalhos, os quais abordam, prioritariamente, o uso de teorias de enfermagem na sistematização da assistência ao hipertenso. É válido ressaltar que apenas sete estados foram citados como local de realização dos estudos, do total de 27 estados brasileiros. Alguns estudos, analisados apenas pelos resumos, não revelavam seu local de realização.

Esses resultados alertam para a necessidade de desenvolvimento de pesquisas sobre o tema em questão em todas as regiões do país, haja vista as consequências da condição de cronicidade da hipertensão arterial, bem como o incremento das doenças cardiovasculares no Brasil. A distribuição geográfica da produção científica da enfermagem não é uniforme em nosso país, equiparando-se à localização dos cursos de pós-graduação ${ }^{(9)}$.

O produto e o processo da atividade científica são dependentes da comunicação eficaz e as revistas especializadas são importantes veículos de divulgação do conhecimento científico. Consoante visualizamos no Gráfico 1, o maior número de publicações sobre o tema (13,3\%) foi encontrado em periódicos de enfermagem com classificação Internacional A, bem como em dissertações.

Somando-se o quantitativo de publicações na Revista Latino-americana de Enfermagem, Texto e Contexto e as dissertações, obtemos um total de 12 (39,9\%) estudos. A Revista Latino-Americana foi a primeira a ser indexada e classificada como periódico internacional, e assim como a Revista Texto e Contexto, é uma das poucas com essa classificação, despertando o interesse dos pesquisadores em nelas publicar.

Ainda no Gráfico 1, representando a categoria "outras" temos as revistas Online Brasilian Journal Nursing, Revista da Rede de Enfermagem do Nordeste, Mundo da Saúde, Revista Eletrônica de Enfermagem, Revista Brasileira de Enfermagem, Revista da Escola de Enfermagem da USP, Acta Paulista, Cogitare, Revista de Enfermagem da UERJ e Revista da Sociedade de Cardiologia de São Paulo com apenas uma publicação.

Percebemos, assim, a necessidade de divulgação das pesquisas realizadas, com vistas à transformação social e, no âmbito da Enfermagem, a transformação do cuidado. Tradicionalmente as descobertas científicas ganharão reconhecimento e credibilidade em função de sua publicação em revistas científicas de prestígio(10) $^{(10}$ 


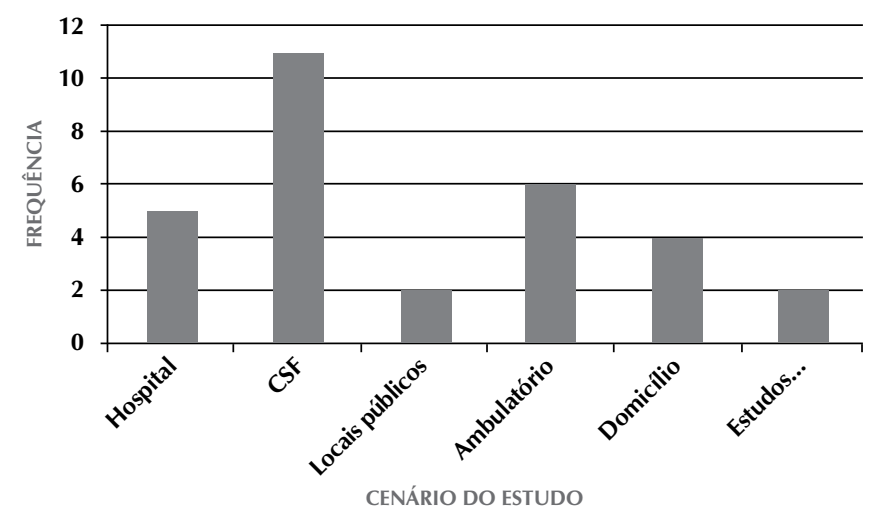

\section{Gráfico 2: Descrição dos estudos segundo cenário da pesquisa. Fortaleza- CE, 2009.}

Quanto ao cenário da pesquisa, constatamos no gráfico 2 a prioridade à atenção primária, uma vez que a maioria dos estudos (36\%) ocorreu em Centros de Saúde da Família (CSF). Em seguida, identificamos os estudos realizados em ambulatórios especializados a nível da atenção secundária, correspondendo a 6 estudos (20\%). Dois $(7 \%)$ configuram-se como estudos reflexivos, não abordando, portanto, local de realização de coleta de dados.

Recomenda-se que de 60 a $80 \%$ dos casos de hipertensão arterial e diabetes mellitus sejam tratados na rede bási$\mathrm{ca}^{(11)}$. Dessa forma, entende-se ser extremamente importante a realização de pesquisas nesses cenários, locais férteis para ações de educação em saúde, objetivando a promoção da saúde e a prevenção de doenças.

Na Atenção Primária à Saúde (APS), a promoção do autocuidado e da adesão ao tratamento deve ser um dos principais objetivos a serem implementados pela estratégia de saúde da família. Tal desenho organizativo atua como porta de entrada e propõe um novo modelo de atenção à saúde baseado nas ações de promoção, prevenção, proteção, recuperação e reabilitação.

No que se refere às práticas de cuidado identificadas, o Quadro 2 traz as temáticas de cuidados de enfermagem identificados nos estudos.

A realização de consultas de enfermagem foi identificada em 17 estudos, sendo uma atividade largamente utilizada em nossa prática assistencial. Nesse contexto, preocupa-nos a abordagem, prioritariamente individual, em detrimento de uma abordagem coletiva, ou seja, que convide membros da família ou outras pessoas importantes à participação e engajamento no tratamento.

Diante da complexidade da práxis da enfermagem, um instrumento metodológico é imprescindível para a realização de um cuidado de qualidade, o qual vem sendo desenvolvido por meio do processo de enfermagem e implementado com a sistematização da assistência de enfermagem. Dentre os estudos analisados, 16 versam sobre a sistematização da assistência de enfermagem, bem como o uso de teorias em sua implementação. As teorias utilizadas foram: Teoria do Autocuidado de Dorothea Orem (sete estudos), Teoria do Alcance de Metas de Imogene King (cinco estudos) e Teoria da Adaptação de Sister Callista Roy (dois estudos). O uso dessas teorias é bem pertinente em situações de cronicidade, uma vez que enfatizam a necessidade do autocuidado, do planejamento de ações conjuntas entre enfermeiro e cliente, e da adaptação do indivíduo a sua doença e tratamento, respectivamente.

No que se refere à utilização da educação em saúde, observamos predomínio de orientações individuais (dez estudos), não ocasionando o impacto desejado na promoção da saúde e da prevenção de doenças. Educação em saúde é definida como quaisquer combinações de experiências de aprendizagem delineadas com vistas a facilitar ações voluntárias conducentes à saúde ${ }^{(12)}$. Por combinação, refere-se à junção de experiências de aprendizagem do cotidiano com intervenções educativas, que devem ser sistematicamente planejadas a possibilitar mudanças comportamentais positivas para a saúde de pessoas, grupos ou comunidades.

Na contramão da idealizada educação em saúde, observa-se uma educação normativa, conteudista, cientificista, enunciada no imperativo, baseada em relação linear entre saber instituído e comportamento. Há um hiato entre discurso e prática pedagógica, bem como a ineficácia da educação em provocar mudanças de comportamento e desenvolver o empowerment $t^{(13)}$.

Vemos, portanto, a necessidade de superação do caráter meramente instrumental, individualista e focado na doença proposto pela educação tradicional tendo em vista a adoção de uma educação libertadora, que desenvolva consciência crítica e instrumentalize indivíduos e comunidades na conquista de sua cidadania e na tomada de decisões ${ }^{(14)}$. Atenta-se para a compreensão da educação em saúde como um momento de construção compartilhada de conhecimentos entre educador e educando.

A temática da visita domiciliária, presente em quatro estudos, dá-se em sua maioria em um contexto de abordagem familiar. A família é um importante sistema de apoio, podendo servir de alicerce ao indivíduo em seu enfrentamento individual

Tabela 2 - Descrição das unidades e sub-unidades temáticas dos estudos sobre cuidado de enfermagem ao cliente com hipertensão. Fortaleza-CE, 2009.

\begin{tabular}{l|l|c}
\hline UNIDADES TEMÁTICAS* & \multicolumn{1}{|c}{ SUBUNIDADES TEMÁTICAS } & f \\
\hline \multirow{2}{*}{ Consultas } & - Abordagem individual & 17 \\
& - Realização de anamnese e exame físico & 2 \\
& - Sistematização da assistência de enfermagem & 16 \\
\hline \multirow{2}{*}{ Educação em saúde } & - Individual no momento da consulta & 10 \\
& - Em grupo & 7 \\
\hline \multirow{2}{*}{ Visitas domiciliárias } & - Abordagem individual & 1 \\
\hline Grupos de autoajuda & - Abordagem familiar & 3 \\
\hline Grupos de atividade física & - Abordagem coletiva & 1 \\
\hline
\end{tabular}

* Alguns estudos apresentaram mais de uma unidade temática. 
cotidiano. Por isso, apontamos a importância da interação enfermeiro-cliente-família.

A realização de atividades coletivas como grupos de autoajuda e de atividades físicas apresentam impactos positivos no tratamento, entretanto, tem sido pouco divulgada na produção científica de enfermagem.

\section{DISCUSSÃO}

Diante da apresentação quantitativa dos trabalhos selecionados, voltamo-nos à sua análise. Uma das principais atividades relatadas nos trabalhos foi a Consulta de Enfermagem. Porém, esta não tem se dado de forma sistemática. A utilização do Processo de Enfermagem dá um caráter profissional à consulta, organizando a abordagem do cliente e definindo a competência da enfermeira. Assim, constatamos que a Consulta de Enfermagem sem sistematização resulta na falta de objetivos próprios e sendo realizada como uma fase preliminar à consulta médica (pré-consulta) ou mesmo complementar (pós-consulta). A enfermeira, por não ter marcos referenciais específicos para a abordagem do cliente, centra a sua consulta em fatores isolados, que representam risco para a situação clínica ${ }^{(15)}$.

Por outro lado, o processo de enfermagem é uma tecnologia que permite realizar, de forma sistemática e dinâmica, um cuidado humanizado, dirigido a resultados positivos e de baixo custo. Possibilita compreender, descrever e/ou explicar como a clientela responde aos problemas de saúde e processos vitais, e determinar que aspectos exigem intervenções de enfermagem ${ }^{(16,17)}$.

A adoção de teorias no cotidiano da enfermagem contribui para a construção do conhecimento científico e para melhor elucidação do real papel do enfermeiro, com reflexo direto no processo de cuidar ${ }^{(18)}$. As teorias desafiam as práticas existentes, uma vez que possibilitam criar novas abordagens e remodelar normas e princípios vigentes.

Para o cuidador, em sua relação com o paciente, deve-se priorizar: interações e conexões positivas, exigindo da enfermeira paciência, harmonia, calma, afetividade e afirmação, aceitação mútua, respeito mútuo e comunicação eficaz.

Esses elementos foram confirmados em outro estudo, quando abordado que o paciente hipertenso freqüenta a consulta de enfermagem por aspectos de ordem emocional. O enfermeiro pode ser identificado como um elemento de confiança no compartilhamento dos problemas e questões de ordem biológica, social, familiar, econômica e emocional ${ }^{(19)}$.

O diagnóstico da Hipertensão Arterial, em geral, faz-se por consulta de outras afecções, muitas vezes já havendo complicações da doença, por queixas diversas, ou seja, de forma casual, o que confirma a necessidade de que a medida da PA seja rotina nas consulta médica e de enfermagem.

Sobre a percepção do cliente hipertenso acerca de sua doença e tratamento, foi constatado que há apreensão moderada do conhecimento dado pelo profissional, referente à prática da caminhada, da dieta hipossódica e do uso correto da medicação. Apesar de informados, referem diferentes motivos para o não atendimento das orientações: comodismo, doenças associadas, pouca segurança, falta de tempo, desgaste físico e mental para realização de exercícios físicos; dificuldades socioeconômicas para a alimentação adequada; dependência química para o fim do tabagismo e etilismo, efeito adverso das drogas para o uso sistemático das medicações.

Em um estudo onde se buscou apreender as representações sociais de mulheres portadoras de hipertensão arterial, dentre as categorias que emergiram, destacou-se a que aponta as explicações das hipertensas sobre o tratamento e controle da doença, evidenciando conhecimentos elaborados e compartilhados socialmente ${ }^{(20)}$. Esses conhecimentos também podem ser confirmados em um estudo com um grupo focal de hipertensos no qual emergiram três categorias, dentre as quais se destacam: "repercussões orgânicas da hipertensão" e "promoção da saúde do hipertenso". Nestas categorias evidenciaram-se conhecimentos acerca dos sinais e sintomas da hipertensão arterial, bem como o tratamento farmacológico e não farmacológico necessários para a prevenção de outras doenças e promoção da saúde ${ }^{(21)}$.

Outro fator importante encontrado foi a relação entre o tempo do tratamento e o uso sistemático das medicações, pois quanto maior o tempo, menor o uso dos remédios, principalmente, nos idosos. Assim, uma avaliação da influência dos medicamentos na qualidade de vida dos hipertensos é imprescindível na busca de alternativas para maior adesão. Assim, a receita ainda é um dos principais fatores do comparecimento dos hipertensos à consulta médica ${ }^{(6)}$.

No mesmo trabalho, evidenciou-se o desconhecimento e a busca de informações por parte dos pacientes. Porém, as condutas profissionais são, em sua predominância, higienodietéticas, exigindo mudanças de hábitos ou estilo de vida. Falta sensibilidade para informar o paciente sobre o que é a Hipertensão, a fim de capacitá-lo a tomar decisões conscientes sobre seu comportamento de busca de saúde.

Há pouca regularidade nas atividades educativas, espaço potencializador no estabelecimento de mudança de comportamento para exercício da cidadania, o que ultrapassa a mera orientação prescritiva. A justificativa para a baixa demanda foi a pouca divulgação, poucos recursos, acomodação e desmotivação.

O processo educativo é uma ação política e social, cujos métodos e técnicas devem favorecer a desalienação, a transformação e a emancipação dos sujeitos envolvidos. Nesse contexto, a educação em saúde não deve ser exclusivamente informativa, normativa, mas deve levar os usuários a refletirem sobre as bases sociais de sua vida, passando a perceber a saúde não mais como uma concessão, e sim, como um direito social defendido por lei ${ }^{(5)}$.

As abordagens educativas existentes têm enfoque na cura, na prevenção de doenças, reproduzindo a assistência queixa-conduta, por meio de prescrições multifatoriais, desenvolvendo interações em nível desigual. Faz-se necessário para o profissional de enfermagem buscar estratégias que estimulem a mudança de comportamento por parte do paciente, pois a adoção apenas de medidas de orientação não é suficiente para adesão eficiente ao tratamento ${ }^{(15)}$.

É consenso afirma que programas estruturados de educação em saúde para adultos portadores de hipertensão arterial levam a melhorias nas condições de saúde destes indivíduos, tanto no 
que se refere aos fatores de risco como para a adesão ao tratamento instituído. Em um estudo onde analisou-se dois artigos que tratavam de intervenções educativas com hipertensos, foi verificado que houve, após a realização de programas educacionais, considerável mudança de comportamento e melhoria da adesão ao tratamento medicamentoso. Reafirmam também as melhorias proporcionadas pela educação em saúde como estratégia junto a pacientes hipertensos adultos. No entanto, também é referido que essas ações não são tão fáceis de serem implementadas, por diversos motivos como o método utilizado, a própria interação com os sujeitos, entre outros ${ }^{(22)}$.

A família também foi abordada nos estudos sobre Hipertensão Arterial, chegando-se à conclusão de que a mesma necessita de informações sobre o processo de adoecimento a fim de ajudar o parente com Hipertensão. Incentivou-se a automonitorização da pressão arterial pelo paciente, necessitando de ajuda da família, principalmente quando se trata de idosos ${ }^{(23)}$.

As doenças crônicas trazem limitações e alterações de estilo de vida de difícil adaptação, mas essas são perfeitamente compatíveis com a manutenção da qualidade de vida do indivíduo ${ }^{(23)}$. Para tanto, é necessário que este seja orientado em relação aos cuidados que deve tomar e os faça de forma consciente, e que a família seja esclarecida a favorecer esta independência, ajudando-o a ter uma vida compatível com sua condição de cronicidade. Além disso, o adulto hipertenso em tratamento contínuo, apesar de conhecer algumas das causas e conseqüências da hipertensão, bem como hábitos saudáveis de vida, subestima os efeitos dessa condição patológica sobre sua função renal.

Constatou-se que nos trabalhos voltados para propostas de atendimento de enfermagem à clientela hipertensa, os grupos melhoraram sua adesão ao tratamento, em relação ao consumo de sal, tabagismo, etilismo, exercícios físicos e estresse. Os pacientes reconhecem suas potencialidades, havendo inclusive a participação da equipe. A implementação de tecnologias relacionais de cuidado comprovou que a ênfase nas relações interpessoais enfermeira/paciente/família foi um dos fatores a propiciar aumento da adesão ao tratamento, mesmo quando a resolução de problemas estruturais do serviço se mostrou inalterada, demonstrando necessidade de cuidar de maneira integral, sistêmica e interativa ${ }^{(7)}$.

Um dos estudos identificados propôs a ação conjunta do enfermeiro, farmacêutico e médico na assistência ao hipertenso, no sentido de prevenir problemas relacionados à medicação. Assim, enfermeiro e farmacêutico trabalhariam na educação referente ao peso, ingestão de sódio, consumo de álcool, atividade física, tabagismo e nas complicações da HA.

Apesar de constatar lacunas na prática, que supririam a baixa adesão existente na clientela hipertensa, as metodologias de cuidado ao cliente hipertenso pelo enfermeiro são diversificadas, possibilitando o uso da criatividade, de aspectos físicos e emocionais de forma a promover a saúde e o bem-estar.

\section{CONCLUSÃO}

Ao escolher esse tema para estudo, acreditamos ser grande o quantitativo de textos que seriam encontrados em virtude da importância do estudo de uma doença tão prevalente e incidente na atualidade. Contudo, consideramos insuficiente a literatura encontrada nas bases de dados no que se refere à operacionalização da prática dos enfermeiros a esses pacientes com vistas à melhoria na qualidade da assistência.

O estudo permitiu visualizar a produção científica do cuidado de enfermagem ao hipertenso, impulsionando a reflexão sobre a necessidade de mais publicações na temática. Trata-se de indivíduos em condição de cronicidade, necessitando de abordagens inovadoras, as quais promovam maior adesão ao tratamento anti-hipertensivo.

É preocupante a forma como foi relatada a realização da Consulta de Enfermagem, ou seja, de forma assistemática, individualizada e ainda centrada no modelo médico hegemônico.

Os hipertensos possuem consciência sobre os hábitos inadequados, porém sentem-se desmotivados às mudanças, em virtude da cronicidade da doença. Desse modo, confirmamos a falta de adesão, conhecimento insuficiente e precariedade no autocuidado, o que é fortalecido pela ausência de sintomas, cujo caráter silencioso, mascara sua gravidade e faz com que os pacientes se acomodem à condição de cronicidade.

Por outro lado, visualizamos possibilidades de mudanças, por meio de condutas que valorizem o outro, seja no ambiente familiar, nas atividades de grupo, ou nas unidades de saúde, promovendo, assim, maior adesão do cliente hipertenso ao tratamento. Para uma ação efetiva na prevenção e no controle da hipertensão, é necessário aliar educação, trabalho e fator social, não em âmbito individual e sim coletivo.

Nesse contexto, esperamos desafiar os enfermeiros assistenciais e pesquisadores a refletirem acerca das práticas de cuidado de enfermagem à clientela hipertensa, no sentido de contribuir para o seu controle e qualidade de vida dos pacientes e de seus familiares.

\section{REFERÊNCIAS}

1. Sociedade Brasileira de Hipertensão $(\mathrm{SBH})$; Sociedade Brasileira de Cardiologia (SBC); Sociedade Brasileira de Nefrologia (SBN). V Diretrizes brasileiras de hipertensão arterial. São Paulo: 2006.

2. Brasil. Hipertensão arterial sistêmica para o Sistema Único de Saúde. Ministério da Saúde, Secretaria de Atenção à Saúde, Departamento de Atenção Básica. - Brasília : Ministério da Saúde, 2006.
3. Araújo GBS, Garcia TR. Adesão ao tratamento anti-hipertensivo: uma análise conceitual. Rev Eletrônica Enferm. 2006;8(2):250-6.

4. Busnello RG, Melchior R, Faccin C, Vettori D, Petter J, Beltrami L. et al. Características associadas ao abandono do acompanhamento de pacientes hipertensos atendidos em um ambulatório de referência. Arq. Bras Cardiol. 2001;76(5):352-4. 
5. Toledo MM, Rodrigues SC, Chiesa AM. Educação em saúde no enfrentametno da hipertensão arterial: uma nova ótica para um velho problema. Texto Contexto Enferm $2007 \mathrm{abr} / \mathrm{mai} ; 16(2): 233-8$.

6. Santos ZMA, Frota MA, Cruz DM, Holanda SDO. Adesão do cliente hipertenso ao tratamento: análise com abordagem interdisciplinar. Texto Contexto Enferm 2005;14(3):332-40.

7. Moreira TMM, Araújo TL. Verificação da eficácia de uma proposta de cuidado para aumento da adesão ao tratamento da hipertensão arterial. Acta Paul. Enferm. 2004;17(3):268-77.

8. Barreto JAE, Moreira RVO. Para além das colunas de Hércules: filosofia e ações de enfermagem. Sobral (CE): Edições UVA, 2004.

9. Carvalho EC. A produção do conhecimento em enfermagem. Rev. Latino-Am. Enfermagem. 1998; 6(1):119-22.

10. Marziale MHP. Produção científica da enfermagem brasileira: a busca pelo impacto internacional. Rev. Latino-Am. Enfermagem. 2005;13(3):285-6.

11. Brasil. Ministério da Saúde. Secretaria de Políticas Públicas. Guia prático do Programa de Saúde da Família. Brasília; 2001.

12. Candeias NMF. Conceitos de educação e de promoção em saúde: mudanças individuais e mudanças organizacionais. Rev Saúde Públ. 1997;31(2):209-13.

13. Grazzinelli MF, Grazzinelli A, Reis DC, Penna CMM. Educação em saúde: conhecimentos, representações sociais e experiências da doença. Cad. Saúde Pública.2005;21(1): 200-206.

14. Meyer DEE, Mello DF, Valadão MM, Ayres JRCM. "Você aprende. A gente ensina? Interrogando relações entre educação e saúde desde a perspectiva da vulnerabilidade". Cad. Saúde Pública. 2006;22(6):1335- 42.
15. Maciel ICF, Araújo TL. Consulta de enfermagem: análise das ações junto a programas de hipertensão arterial, em Fortaleza. Rev. Latino-Am. Enfermagem. 2003; 11(2):207-14

16. Alfaro-Lefreve R. Aplicação do processo de enfermagem: promoção do cuidado colaborativo. trad.: Regina Garcez. $5^{\mathrm{a}}$ ed. Porto Alegre (RS): Artmed, 2005.

17. Garcia TR, Nóbrega MML. Sistematização da assistência de enfermagem: reflexões sobre o processo. In: $52^{\circ}$ Congresso Brasileiro de Enfermagem, apresentado na Mesa Redonda "A sistematização da assistência de enfermagem: o processo e a experiência". Recife/Olinda-PE, 2000.

18. Oliveira TC, Lopes MVO, Araujo TL. Modo fisiológico do modelo de adaptação de Sister Callista Roy: análise reflexiva de Meleis. Online Brazilian Journal of Nursing, 2006; 5(1).

19. Lyra Jr DP, Amaral RT, Veiga EV, Cárnio EC, Nogueira MS, Pelá IR. A farmacoterapia do idoso: revisão sobre a abordagem multiprofissional no controle da hipertensão arterial sistêmica. Rev. Latino-Am. Enfermagem. 2006;14(3):435-41.

20. Silva MEDC, et. al. As representações sociais de mulheres portadoras de Hipertensão Arterial. Rev Bras Enferm 2008;61(4):500-7.

21. Orsolin C, Rufatto C, Zambonato RX, Fortes VLF, Pomati DM. Cuidando do ser humano hipertenso e protegendo sua função renal. Rev. Bras. Enferm, Brasília. 2005 maio/ jun; 58(3).

22. Chaves ES, Lúcio IML, Araújo TL, Damasceno MMC. Eficácia de programas de educação para adultos portadores de hipertensão arterial. Rev Bras Enferm 2006;59(4).

23. Moreira TMM, Araújo TL, Pagliuca LMF. Alcance da Teoria de King junto a famílias de pessoas portadores de hipertensão arterial sistêmica. Rev. Gaúcha Enferm. 2001 jan; 22(1):74-89. 